\title{
Microscope-assisted coblation tonsillectomy among paediatric patients - our experiences at an Indian teaching hospital
}

\author{
Santosh Kumar Swain', Tushar Kanti Ghosh², Sampada Munjal', Jatindra Nath Mohanty ${ }^{3}$ \\ 'Department of Otorhinolaryngology, IMS and SUM Hospital, Siksha "O" Anusandhan University, K8, Kalinganagar, \\ Bhubaneswar, Odisha, India \\ ${ }^{2}$ Ghosh ENT Foundation, Kolkata, India \\ ${ }^{3}$ Medical Research Laboratory, IMS and SUM Hospital, Siksha "O" Anusandhan University, K8, Kalinganagar, Bhubaneswar, \\ Odisha, India
}

\section{ABSTRACT}

Introduction: Tonsillectomy is an established treatment option for chronic tonsillitis or sleep-disordered breathing defect in the paediatric age group.

Aim of the study: A retrospective study was done to compare surgical parameters such as intra-operative bleeding, duration of surgery, post-operative bleeding, postoperative pain, and hospital stay, in paediatric patients undergoing coblation tonsillectomy with or without assistance of an operating microscope.

Material and methods: Data regarding intra-operative bleeding, postoperative pain, post-operative bleeding, and hospital stay were collected from the medical records of the paediatric patients undergoing coblation tonsillectomy with or without microscope assistance during the preceding five years, from January 2014 to February 2019.

Results: A total of 394 children participated in this study. Out of the 394, 212 paediatric patients underwent coblation tonsillectomy under an operating microscope, and 182 underwent coblation tonsillectomy under a headlight. There was no significant difference between the two groups in terms of duration of surgery among coblation tonsillectomy with or without a microscope. The incidence of primary haemorrhage, reactionary haemorrhage, and secondary haemorrhage were significantly reduced in the case of microscope-assisted coblation tonsillectomy than direct tonsillectomy with the help of just a headlight. Pain scores and hospital stay are significantly reduced with microscope-assisted coblation tonsillectomy.

Conclusions: Microscope-assisted coblation tonsillectomy reduces the intra-operative and post-operative haemorrhage, post-operative pain, and length of hospital stay, thus helping in early improvement of the patient. The overall advantages of microscope-assisted coblation tonsillectomy compared to coblation tonsillectomy under just a headlight are the following: reduced intra- and post-operative bleeding, better safety, precision of tonsil removal, and less injury to the adjacent tissues. For these reasons, microscope-assisted coblation tonsillectomy in children is recommended as a safe and effective method for tonsillectomy.

\section{KEY WORDS:}

tonsillectomy, paediatric patients, coblation, microscope assisted.

\section{ADDRESS FOR CORRESPONDENCE:}

Santosh Kumar Swain, Department of Otorhinolaryngology, IMS and SUM Hospital, Siksha "O” Anusandhan University, K8, Kalinganagar, 751003 Bhubaneswar, Odisha, India, ORCID: 0000-0001-7933-4414,

e-mail: santoshvoltaire@yahoo.co.in 


\section{INTRODUCTION}

Tonsillectomy is one of the commonest surgical procedures performed among paediatric patients. This procedure is done to remove palatine tonsils by dissection at the peritonsillar space. It is often indicated in cases of recurrent tonsillitis as well as tonsillar hypertrophy causing sleep disorders [1]. Palatine tonsils are lymphoid tissues located at the oropharynx within the tonsillar fossa. In the paediatric age group, palatine tonsils play a role in the defence mechanism and immunology. Secretions of antibodies such as IgA play an important role in the mucosal defence mechanism. In the case of repeated infection of the tonsils, the protective mechanisms of tonsils fail and cause sore throat, fever, and other complications for which tonsillectomy is necessary [2]. Tonsillectomy is a common surgical procedure faced during otolaryngological practice. Tonsillectomy is a surgical procedure in which post-operative pain, haemorrhage, and other complications are seen. Post-tonsillectomy haemorrhage is not an uncommon event, and its prevalence varies widely, ranging from $2.7 \%$ to $15.9 \%$ [3]. Different surgical techniques for tonsillectomy have been successfully attempted over the years, including the guillotine method, diathermy, and dissection, as well as more recent methods like laser, ultrasonic, and coblation. Coblation tonsillectomy is one of the more recent techniques described in the medical literature. The postoperative complications of tonsillectomy are pain in the throat, haemorrhage, otalgia, and fever [4]. Coblation is an advanced technology currently used for tonsillectomy. This technology combines radiofrequency energy and saline to create a plasma field. Coblation never removes tissue by heating or burning [5]. Here we study the details of microscopeassisted coblation tonsillectomy, including complications like intra-operative haemorrhage and pain in paediatric patients.

\section{MATERIAL AND METHODS}

This is an observational, descriptive, and retrospective study carried out at a tertiary care teaching hospital in eastern India. A total of 394 paediatric patients participated in this study. Out of the 394 patients, 212 patients underwent coblation tonsillectomy under a microscope over the period of five years between January 2014 and February 2019. This study was approved by our Institutional Ethics Committee (IEC). There were 182 patients who underwent coblation tonsillectomy under a headlight. All children selected for tonsillectomy had recurrent throat infections and had been diagnosed with chronic tonsillitis. Children with bleeding disorders, anaemia, acute infections (history of tonsillitis within three weeks prior to surgery), anaesthetic risk, and uncontrolled medical illness were excluded from the study. General anaesthesia was preferred in the paediatric patients. A Boyle's Davis mouth gag was used in all cases in an extended neck position (Fig. 1). In total 212 children underwent tonsillectomy by coblation under an operating microscope. All the surgery was performed by senior authors. The coblation tonsillectomy was done by using the Smith and Nephew ENT Coblator II surgery system with EVac 70 plasma wands (Arthro Care Corporation, Sunnyvale, CA). Power settings were standardised at seven for ablation and three for coagulation. Surgery was done under operating microscope. The operative field was better visualised under microscopic view (Fig. 2). Blood loss during surgery was documented. Duration of surgery in each case was also documented. A telephonic record sheet was maintained for documenting the data corresponding to pain, and reactionary and secondary haemorrhage on the $3^{\text {rd }}, 7^{\text {th }}$, and $14^{\text {th }}$ day. Parents of the children were advised to keep a pain diary and record details on a daily basis for two weeks after surgery including the following information: 1) visual analogue score for pain of $0-10$, where 0 represents no pain, 1-3 represents mild pain, 4-7 represents moderate pain, and 10 indicates extreme pain; 2 ) number of analgesic tablets taken for that day; 3 ) ability to take normal food; 4) ability to return to perform normal activities; and 5) experiencing pain during swallowing. A pain score of $\leq 3$ was set as the cut-off value considered as the level of pain that would not affect quality of life. The pain score was calculated at rest and during swallowing daily

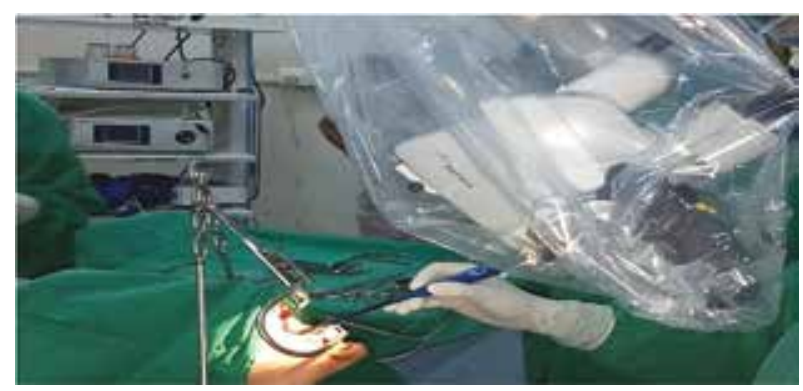

FIGURE 1. Position of the patient during microscope-assisted coblation tonsillectomy

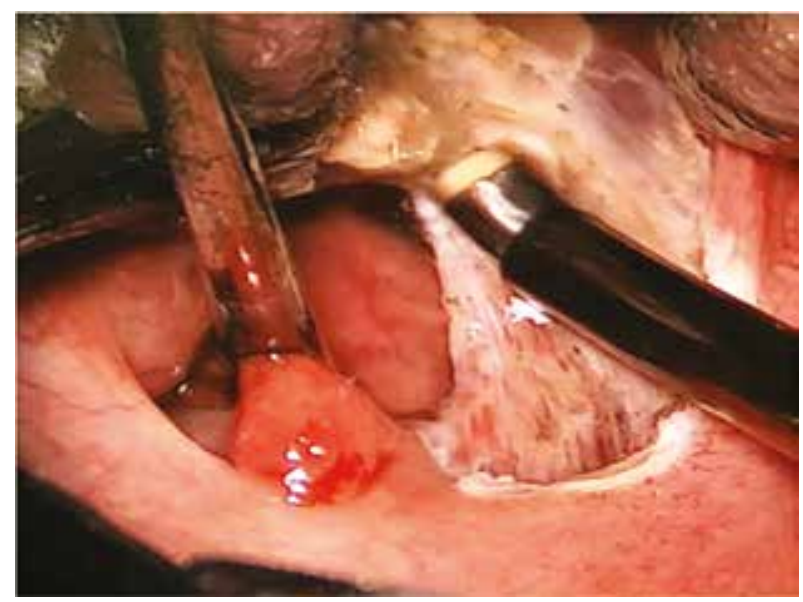

FIGURE 2. Microscopic intra-operative picture of coblation tonsillectomy 
TABLE 1. Clinical profile of the patients who underwent microscopic coblation tonsillectomy

\begin{tabular}{|l|c|c|}
\hline Parameters & $\begin{array}{c}\text { Number of patients } \\
(n=212)\end{array}$ & Percentage \\
\hline $\begin{array}{l}\text { Sex } \\
\text { Male }\end{array}$ & 126 & 59.43 \\
\hline Female & 86 & 40.56 \\
\hline Age & & \\
\hline 3 to 10 years & 121 & 57.07 \\
\hline 11 to 16 years & 91 & 42.92 \\
\hline
\end{tabular}

TABLE 2. Clinical profile of the patients who underwent coblation tonsillectomy under a headlight

\begin{tabular}{|l|c|c|}
\hline Parameters & $\begin{array}{c}\text { Number of patients } \\
(n=182)\end{array}$ & Percentage \\
\hline $\begin{array}{l}\text { Sex } \\
\text { Male }\end{array}$ & 98 & 53.84 \\
\hline Female & 84 & 46.15 \\
\hline Age & & \\
\hline 3 to 10 years & 101 & 55.49 \\
\hline 11 to 16 years & 81 & 44.50 \\
\hline
\end{tabular}

at the same time for first seven post-operative days by face-to-face or telephone enquiry. Data were calculated as mean \pm SD. Intra-operative blood loss, duration of surgery, secondary haemorrhage, post-operative pain, and post-operative hospital stay were analysed by using Student's independent two-sample $t$-test. A $p$-value of $<0.05$ was considered statistically significant. All the statistical calculations were carried out with SPSS software (version 15, SPSS Inc., Chicago, IL, USA).

\section{RESULTS}

This is a retrospective study in which 394 paediatric patients participated. Out of the 394, 212 patients underwent coblation tonsillectomy under an operating microscope, and 182 underwent coblation tonsillectomy under a headlight. Out of the 212 children who underwent microscope-assisted coblation tonsillectomy, 126 were males and 86 were females, with a mean age of 7.45 years and age range 3 to 16 years. Patients were divided into two groups according to the use of either the operating microscope or a headlight. Tonsillectomy was performed using a headlight among 182 children 98 males and 84 females, with mean age of 8.24 years. Microscope-assisted coblation tonsillectomy was done by using an operating microscope among 126 male patients and 86 female patients in a tertiary care teaching hospital of eastern India. Patient data were carefully recorded, including intra-operative blood loss, postoperative pain, and duration of postoperative hospital stay. The intra-operative blood loss was recorded by a staff nurse as the volume of fluid obtained via suction tube minus the volume of saline flush. Detail clinical data are shown in Table 1 and 2. There were no significant between-group differences in terms of duration of surgery, but there was significant difference between the two groups in terms of primary and postoperative haemorrhage (Table 3 ). There was also significance lower post-operative pain and shorter hospital stay in patients who underwent tonsillectomy under an operating microscope $(p<0.05)$ (Table 3$)$. In this study we performed microscope-assisted coblation tonsillectomy in paediatric patients, and on the basis of our experiences it was shown that this technique allows minimally invasive tonsillectomy with less or no damage to surrounding soft tissue, minimal or no blood loss, and minimal post-operative complications.

\section{DISCUSSION}

Tonsillectomy is one of the most common surgical procedures performed by otolaryngologists during their practice period. Paediatric tonsillectomy continues to be the commonest paediatric surgical procedure. There have been several techniques, for example by Comelius Cesus

TABLE 3. Clinical parameters of the patients who underwent coblation tonsillectomy without or with microscope assistance

\begin{tabular}{|l|c|c|c|}
\hline Patient parameters & $\begin{array}{c}\text { Tonsillectomy done } \\
\text { under headlight } \\
(n=182)\end{array}$ & $\begin{array}{c}\text { Tonsillectomy done under } \\
\text { operating microscope } \\
(n=212)\end{array}$ & $p$-value \\
\hline Duration of surgery (in minutes) & $24.2 \pm 3$ & $25.3 \pm 2$ & $<$ \\
\hline $\begin{array}{l}\text { Primary haemorrhage (intra-operative blood } \\
\text { loss in ml) }\end{array}$ & 13 & 5 & $<0.001$ \\
\hline Secondary haemorrhage in ml & 9 & 3 & $<0.001$ \\
\hline Post-operative hospital stay & 2.5 days & 1.4 days & $<0.001$ \\
\hline \begin{tabular}{l} 
Post-operative pain (time to VAS pain score) \\
\hline During rest
\end{tabular} & 6.43 & 3.21 & $<0.001$ \\
\hline During swallowing & 7.21 & 3.91 & \\
\hline
\end{tabular}


in the first century AD and Paul of Aegina in $625 \mathrm{AD}$ [6]. Despite the different range of techniques currently used for tonsillectomy, including guillotine, blunt dissection, diathermy, or laser and the use of several analgesics, post-operative pain and haemorrhage are the major side effects of this operation [7]. In addition to the above techniques, there are several newer methods, such as $\mathrm{CO}_{2}$ lasers, harmonic scalpels using ultrasonic energy, micro-debriders, and coblation using bipolar radiofrequency ablation [8]. Intra-operative and post-operative blood loss is dependent on several factors including the technique or instruments used for the procedure, the speed of the surgery, and the experience of the surgeons, all of which are potential confounding factors affecting the outcome of the tonsillectomy. Very few surgeons perform tonsillectomy under microscope vision [9]. Tonsillectomy is often regarded as a simple surgery, and surgeons often are not bothered about further optimisation and development in this field. The use of the coblation technique in tonsillectomy has added several advantages in comparison to alternative forms of surgery. Coblation tonsillectomy has the important advantage of limiting the thermal injury in comparison to other techniques used for tonsillectomy. The term coblation is derived from the word "controlled ablation", which means a non-heat-driven process for soft tissue dissociation by use of bipolar radiofrequency energy to excite electrolytes in a conductive medium such as saline solution [10].

Coblation technique uses bipolar radiofrequency energy that generates a field of ionised sodium molecules, which ablates and coagulates soft tissue without any thermal injury. Conductive saline solution is kept in the gap between the device tip and the soft tissue and converted into an ionised plasma layer. When this plasma layer touches the tissue, there is enough energy to break the molecular bonds, leading to molecular dissociation. This effect occurs at temperatures of about $40^{\circ} \mathrm{C}$ to $70^{\circ} \mathrm{C}$ thus reducing thermal damage to the tissues [11]. Post-operative pain is an important factor, which limits the recovery from a surgical procedure. Inadequate pain relief can delay and prevent hospital discharge [12]. Telephone follow-up for post-operative pain is usually done on the fourth, seventh, and $15^{\text {th }}$ day because it often coincides with the key stages in the evolution of the post-tonsillectomy inflammatory process. The maximum inflammation of the tonsillectomy wound produced in the third and fifth days of the post-operative period, whereas the separation of the fibrin clot forms around the $7^{\text {th }}$ day after the surgery [13]. Similarly, the appearance of the haemorrhagic process on the $14^{\text {th }}$ day of the post-operative period in some cases made patient follow-up during the second week appropriate.

The important advantages of tonsillectomy under an operating microscope over direct vision are the ability to visualise the detailed anatomy of the extra-capsular loose soft tissues of the tonsil. In addition to good visualisation, students and trainees can see directly the surgical steps through a monitor or observer eye piece. Although many surgeons have a microscope, the majority perform tonsillectomies under direct vision. Many surgeons carry out tonsillectomies under a headlamp or surgical loupe although the lighting and magnifications of the surgical field are inferior to those seen under an operating microscope. In comparison to headlight and loupe, the magnification of the operating microscope can be adjusted at any point during surgery. The microscope is adjusted to $3 \times$ at the initial part of the surgery, and it can be increased to $6-8 \times$ for precise separation of the tonsil from the tonsillar bed and blood vessels. The visualisation and axes of the light are usually parallel, providing good illumination and visualisation of the operating or surgical site. Moreover, the operative microscope prevents the field of view from moving because it has a mechanical arm bracket. These features of the operative microscope enhance the visualisation of the tonsillar bed. Operative microscope provides better quality of brightness, colour, and clarity of light than do headlight and loupes. It can also be connected to an image display system to provide better quality teaching to the trainees.

During the intra-operative time, the ablation mode with a plasma wand is performed only for loose soft tissue between pharyngeal muscles and the capsule of the tonsil to avoid direct damage to the pharyngeal muscles during microscope-assisted coblation tonsillectomy. In comparison to coblation tonsillectomy without assistance of a microscope, the capsule of the tonsils can be easily distinguished along with fine blood vessels over it. The nourishing blood vessels entering the tonsils are only occluded during this surgery. Injury to the paratonsillar veins and tonsillar arteries is effectively avoided by use of the operating microscope. In our cases, injury to the paratonsillar vein (external palatine vein) is an important and major cause of bleeding during surgery by conventional method. Use of an operating microscope helps to distinguish and occlude these veins over the capsule of the tonsils, limiting blood loss. Microscope-assisted coblation tonsillectomy is an improvement over all other techniques used for tonsillectomy in the past with respect to damage to the soft tissue at the surgical field, including blood vessels [14]. Use of the operating microscope allows not only better visualisation of the surgical field but also a lower pole of the tonsils, so there is no residual lower pole tissue, which can cause future infection and hypertrophy [15]. Use of the operating microscope provides better representation of the brightness, colour, and clarity of the operative field than a headlight or loupe, and it can be connected to an image display system to facilitate the teaching of students [16]. The primary advantage of microscope-assisted coblation tonsillectomy is the ability to observe the detailed anatomy of the extra-capsular loose tissues in the tonsillar bed. In addition to the magnified view of the surgical field, the surgical assistants and students can directly see the surgical steps through external or secondary mirror. Microscope-assist- 
ed tonsillectomy is an effective tool for educating students and young surgeons and minimising complications. This study analyses the intra-operative haemorrhage, surgical duration, postoperative pain, reactionary haemorrhage, secondary haemorrhage, and length of hospital stay after surgery in patients undergoing microscope-assisted coblation tonsillectomy. This study has some advantages: only paediatric cases were analysed, and a large number of children were included.

\section{CONCLUSIONS}

Microscope-assisted coblation tonsillectomy allows minimally invasive intervention, reduced intra-operative blood loss, shorter duration of surgery, less postoperative pain, reduced hospital stay, and less chance of reactionary and secondary haemorrhage in comparison to conventional tonsillectomy. The use of an operating microscope does not simply enhance the field of vision, but also offers an innovative change to the surgical approach. Overall advantages of microscope-assisted coblation tonsillectomy compared to coblation tonsillectomy under headlight are reduced intra- and post-operative bleeding, better safety, precision of tonsil removal, and less injury to the adjacent tissues. For these reasons, microscope-assisted coblation tonsillectomy in children is advised as a safe and effective method.

\section{DISCLOSURE}

The authors declare no conflict of interest.

\section{REFERENCES}

1. Kemal O. Harmonic scalpel versus bipolar tonsillectomy: a double-blind clinical trial. Eur Arch Otorhinolaryngol 2012; 269: 1533 1536.

2. Sasindran V, Mathew N, Shabna AK, Harikrishan B. Comparison of Coblation Tonsil-lectomy vs Dissection Tonsillectomy. Int J Otolaryngol Head Neck Surg 2019; 8: 49-60.

3. Benninger M, Walner D. Coblation: improving outcomes for children following adenotonsillectomy. Clin Cornerstone 2007; 9: 13-23.

4. Meybodian M, Dadgarnia M, Baradaranfar M, et al. Effect of cold diet and diet at room temperature on post-tonsillectomy pain in children. Iran J Otolaryngol 2019; 25: 81-86.

5. Lin C, Thung AK, Jatana KR, et al. Impact of coblation versus electrocautery on acute post-operative outcomes in pediatric tonsillectomy. Laryngoscope Investig Otolaryngol 2019; 4: 154-159.

6. Walner DL, Parker NP, Miller RP. Past and present instrument use in pediatric adenotonsillectomy. Otolaryngol Head Neck Surg 2007; 137: 49-53

7. Temple RH, Timms MS. Paediatric coblation tonsillectomy. Int J Pediatr Otorhinolaryngol 2001; 61: 195-198.

8. Noordzij JP, Affleck BD. Coblation versus unipolar electrocautery tonsillectomy: a prospective, randomized, single-blind study in adult patients. Laryngoscope 2006; 116: 1303-1309.

9. Belloso A, Chidambaram A, Morar P, et al. Coblation tonsillectomy versus dissection tonsillectomy: postoperative hemorrhage. Laryngoscope 2003; 113: 2010-2013.
10. Leong AT, Ganhasan S, Sun PL, et al. PEAK plasma blade versus monopolar electrocautery tonsillectomy in adults: A prospective double blinded randomized controlled trial. Am J Otolaryngol 2019 March 18 [Epub ahead of print].

11. Temple RH, Timms MS. Paediatric coblation tonsillectomy. Int J Pediatr Otorhinolaryngol 2001; 61: 195-198.

12. Thung AK, Elmaraghy CA, Barry ND, et al. Double blind randomized placebo controlled trial of single dose intravenous acetaminophen for pain associated with adenotonsillectomy in pediatric patients with sleep disordered breathing. J Pediatr Pharmacol Ther 2017; 22: 344-351.

13. Isaacson G. Tonsillectomy healing. Ann Otol Rhinol Laryngol 2012; 121: 645-649.

14. Magdy EA, Elwany S, el-Daly AS, et al. Coblation tonsillectomy: a prospective, double-blind, randomised, clinical and histopathological comparison with dissection- ligation, monopolar electrocautery and laser tonsillectomies. J Laryngol Otol 2008; 122: 282-290.

15. Pang Y, Gong J, Huang J, et al. Coblation tonsillectomy under surgical microscopy: A retrospective study. J Int Med Res 2016; 44: 923-930.

16. Yi Wong DJ, Paddle P. Harmonic scalpel versus other techniques for tonsillectomy: a systematic review and meta-analysis. Aust J Otolaryngol 2019; 2: 3. 